\title{
GPS network observation of traveling ionospheric disturbances following the Chelyabinsk meteorite blast
}

\author{
Feng Ding ${ }^{1,2}$, Tian Mao ${ }^{3}$, Lianhuan $\mathrm{Hu}^{1,2}$, Baiqi Ning ${ }^{1,2}$, Weixing Wan ${ }^{1,2}$, and Yungang Wang ${ }^{3}$ \\ ${ }^{1}$ Key Laboratory of Earth and Planetary Physics, Institute of Geology and Geophysics, \\ Chinese Academy of Sciences, Beijing, China \\ ${ }^{2}$ Beijing National Observatory of Space Environment, Institute of Geology and Geophysics, \\ Chinese Academy of Sciences, Beijing, China \\ ${ }^{3}$ National Satellite Meteorological Center, China Meteorological Administration, \\ Beijing, China \\ Correspondence to: Feng Ding (dingf@mail.iggcas.ac.cn)
}

Received: 25 June 2016 - Revised: 26 October 2016 - Accepted: 31 October 2016 - Published: 17 November 2016

\begin{abstract}
We use the Global Positioning System (GPS) network in northwest China and central Asia to monitor traveling ionospheric disturbances (TIDs), which were possibly excited by the large meteorite blast over Chelyabinsk, Russia, on 15 February 2013. Two TIDs were observed. The first TID was observed 13 min after the blast within a range of 270 $600 \mathrm{~km}$ from the blast site. It propagated radially from the blast site with a mean velocity and period of $369 \mathrm{~m} \mathrm{~s}^{-1}$ and $12 \mathrm{~min}$, respectively. The second TID was found in northwest China, $1.5 \mathrm{~h}$ after the time of the blast, at $\sim 2500-3100 \mathrm{~km}$ from the blast site. This latter TID propagated southeastward with a velocity and period of $410 \mathrm{~m} \mathrm{~s}^{-1}$ and $23 \mathrm{~min}$, respectively. Severe dissipation of the perturbation total electronic content (TEC) amplitude was observed. Any TIDs propagating in a global range was not found after the meteorite blast. Features of TIDs were compared with those excited by early nuclear explosion tests. It is inferred from our analysis that the energy release of the Chelyabinsk meteorite blast may not be large enough to excite such ionospheric disturbances in a global range as some nuclear explosions did.
\end{abstract}

Keywords. Ionosphere (ionospheric disturbances)

\section{Introduction}

On 15 February 2013, a large meteorite entered the atmosphere near the city of Chelyabinsk, Russia $\left(54.80^{\circ} \mathrm{N}\right.$, $\left.61.10^{\circ} \mathrm{E}\right)$. According to calibrated video observations, the meteorite was characterized by a diameter of $\sim 19.8 \mathrm{~m}$ and a mass of $\sim 1.3 \times 10^{7} \mathrm{~kg}$ (Popova et al., 2013). It was first observed at an altitude of $97 \mathrm{~km}$, moving hypersonically towards the northwest at a shallow entry angle (Borovička et al., 2013). It began to fragment at an altitude of $83 \mathrm{~km}$ under the combined stresses of heating and air drag. The meteorite's peak blast occurred when it reached an altitude of $\sim 30 \mathrm{~km}$ at 03:20 UT, with an energy equivalent to $\sim 500 \mathrm{kt}$ of trinitrotoluene (TNT; Borovička et al., 2013). This was the most energetic meteorite blast on Earth since the 1908 Tunguska event.

Following the Chelyabinsk meteorite blast, infrasound perturbations in air pressure were observed by ground-based infrasound sensors across the entire globe (Brown, 2013). The propagation of this long-distance infrasound-wave is probably driven by acoustic waveguides below an altitude of $40 \mathrm{~km}$ (Pichon et al., 2013). At much higher altitudes, ionospheric disturbances caused by the meteorite blast were observed by the backscatter radar at the ARTI observatory, which is located some $200 \mathrm{~km}$ from the blast site (Berngardt et al., 2013). The radar observed a series of perturbations in the F-layer electron density, which propagated with velocities of 250,400 and $800 \mathrm{~m} \mathrm{~s}^{-1}$. Ionospheric disturbances that occurred after the meteorite blast were also seen in total electronic content (TEC) data observed by the ARTU Global Positioning System (GPS) station, which is located at $242 \mathrm{~km}$ from the blast site (Gokhberg et al., 2013). Recently, Yang et al. (2014) used the global GPS network to observe the ionospheric disturbances that occurred following the meteorite's descent. They recorded three types of disturbances 
that may be associated with the meteorite blast: high-speed ionospheric perturbations near the blast site, low-speed disturbances within $300-1500 \mathrm{~km}$ from the blast site and highspeed disturbances in North America. However, due to a discontinuity of the GPS networks in different continents, the study of such global-scale propagation of ionospheric disturbances is still ongoing.

In this paper, we used the dense GPS network coverage in central Asia and northwest China to monitor propagation features of the traveling ionospheric disturbances following the Chelyabinsk meteorite blast. The Chinese network was operational within a horizontal range of at least $\sim 3000 \mathrm{~km}$ from the blast site. Because there are few ionospheric stations in the near-field area of Chelyabinsk, the GPS network in northwest China appears to be the nearest dense observational network suitable for observations of the ionospheric effects associated with the meteorite blast.

\section{Observations}

TEC data from 75 GPS stations were used in our study. A total of 69 stations were located in northwest China and belonged to the GPS network of the Chinese Meteorological Administration, and another six stations belonging to International Global Navigation Satellite System (GNSS) Service (IGS). Figure 1 shows the geometry of the observations. All GPS stations recorded the carrier phase and pseudorange measured at two frequencies $\left(f_{1}=1575.42 \mathrm{MHz} ; f_{2}=\right.$ $1227.60 \mathrm{MHz}$ ) with a time resolution of $30 \mathrm{~s}$. Based on this information, we calculated the slant TEC for every GPS satellite-receiver pair and converted it to the corresponding vertical TEC. Cycle slips and instrumental bias were corrected by reference to data from global ionospheric maps (Noll, 2012). Next, we obtained the perturbation TEC by subtracting the $30 \mathrm{~min}$ running average from the TEC series.

Figure 2 shows the ionospheric TEC disturbances recorded at the ARTU GPS station, which is the GPS station nearest to the meteorite blast site. Approximately $13 \mathrm{~min}$ after the blast, at 03:33 UT, obvious disturbances appeared in the TEC series for satellite PRN 15, 18, 26 and 29 (see Fig. 2a). The perturbations lasted $\sim 37$ min until 04:10UT. The same disturbances were not noticeable for other satellites (i.e., PRN 16 and 21). As seen from the perturbation TEC series (see Fig. 2b), the perturbation was characterized by an "N"-shaped shock front with a maximum amplitude of $0.35 \mathrm{TECU}$ (total electron content unit, $1 \mathrm{TECU}=10^{16} \mathrm{elec}$ trons $\mathrm{m}^{-2}$ ) and an average period of $12 \mathrm{~min}$. Figure $2 \mathrm{c}$ indicates that the traveling ionospheric disturbance (TID) propagated radially from the blast site. The TEC perturbations occurred at $270-600 \mathrm{~km}$ from the blast site (see Fig. 2c). The horizontal propagation velocities, as estimated by dividing the propagation distances by the relevant time intervals, vary from 341 to $412 \mathrm{~m} \mathrm{~s}^{-1}$, with a mean velocity of $369 \mathrm{~m} \mathrm{~s}^{-1}$.

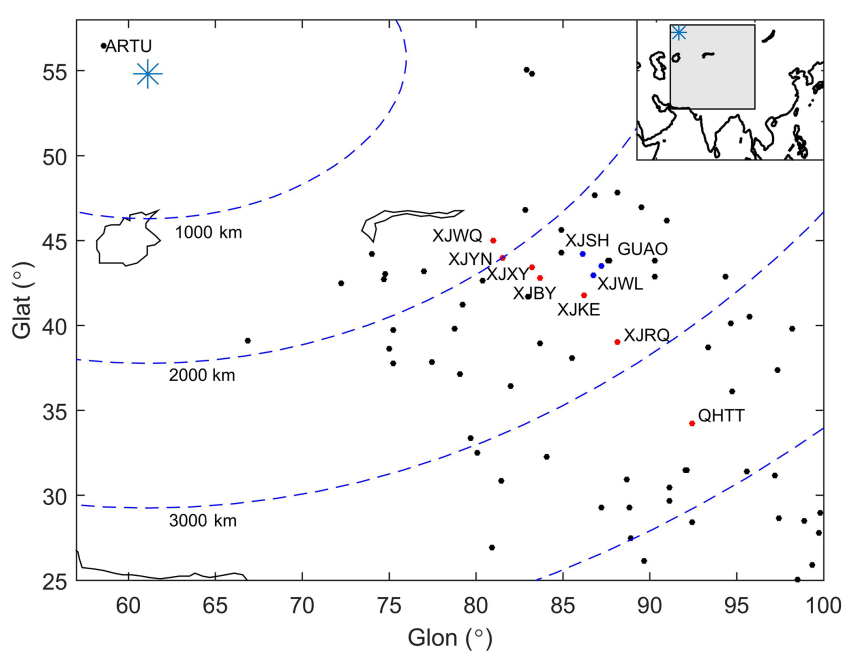

Figure 1. Geometry of the observations. The asterisk marks the location of the meteorite blast site over Chelyabinsk, Russia $\left(54.80^{\circ} \mathrm{N}, 61.10^{\circ} \mathrm{E}\right)$. The dots represent locations of the GPS sites. Blue curves represent the contours of distances from the meteorite blast site. The distances were calculated based on the arcs on the surface of the Earth. The shaded area in the sub-plot on the upperright side indicates the location of the observed area in the map of Asia.

A second TID was observed using the GPS network in northwest China. It is seen in Fig. 3a that traveling ionospheric perturbations occurred during the period 04:4005:30 UT and are characterized by a TEC amplitude of up to $0.6 \mathrm{TECU}$ and an average period of $23 \mathrm{~min}$. Because of the difference in periods, this TID appears to be different from that observed by ARTU. An obvious time delay among the series is seen in Fig. 3a, indicating that the perturbations were propagating away from the blast site. The perturbation amplitude decreased from 0.6 TECU at the XJWQ station to 0.3 TECU at XJKE. This implies severe dissipation of the perturbation amplitude during the propagation of the wave. The perturbation is not noticeable at XJRQ and QHTT. This indicates that the TID did not reach the observational range of them. It is noted from Fig. 3a that some perturbations occurred before 04:40 UT. These perturbations were not identified as a TID event, because there is no regular phase propagation among the TEC series from different stations. Figure $3 \mathrm{~b}$ shows that two phase fronts appeared in the distanceUT plane, which were observed at $\sim 2500-3100 \mathrm{~km}$ from the meteorite blast site. The TID's propagation velocity measured by calculating the slope of the phase fronts is on average $410 \mathrm{~m} \mathrm{~s}^{-1}$.

Figure 4 presents temporal variations of the propagation azimuth, horizontal phase velocity, and period for the second TID we observed in China. Temporal variations of the parameters were obtained by applying a multi-channel maximum entropy method (MMEM) to the TEC time series observed by three Chinese GPS stations: XJSH $\left(44.2^{\circ} \mathrm{N}\right.$, 

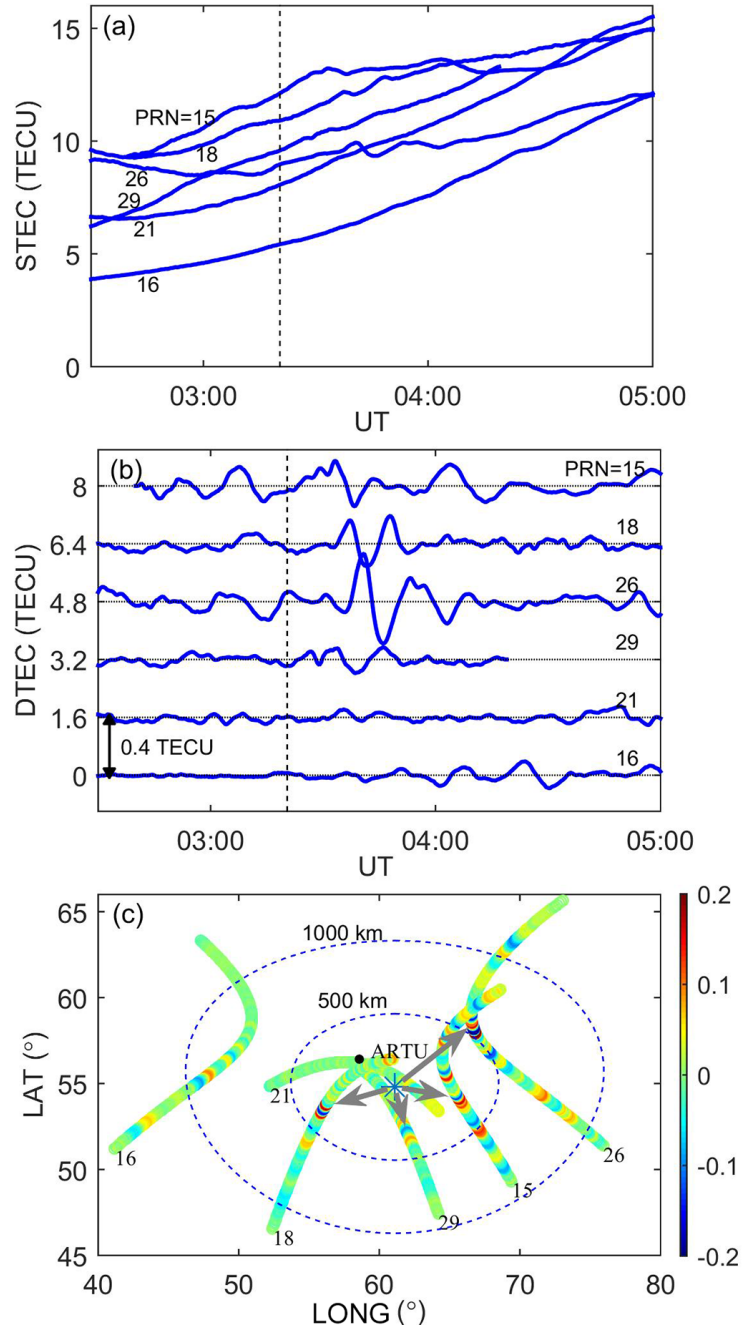

Figure 2. Ionospheric disturbances recorded at the ARTU GPS station on 15 February 2013. (a) Temporal TEC variation. (b) Temporal variation of the TEC perturbation. (c) Spatial distribution of the TEC perturbation. The GPS satellite PRNs corresponding to all TEC series are marked near each series. The vertical dotted lines in panels (a) and (b) mark the time of the meteorite blast (03:20 UT). Curves in Panel (c) indicate the trajectories of the ionospheric pierce points, with the contours representing the amplitude of perturbation in TECUs. The asterisk in panel (c) marks the site of the meteorite blast. Grey lines connect the blast site and the sites where TEC perturbations were recorded, with arrows denoting the propagation directions.

86. $\left.1^{\circ} \mathrm{E}\right)$, GUAO $\left(43.5^{\circ} \mathrm{N}, 87.2^{\circ} \mathrm{E}\right)$, and XJWL $\left(42.9^{\circ} \mathrm{N}\right.$, $\left.86.7^{\circ} \mathrm{E}\right)$. The locations of these stations are marked as blue dots in Fig. 1. MMEM is a statistical spectral analysis technique, which can generate superior-resolution spectral estimates of any measure from a given system by autoregressive fitting. For a detailed description of MMEM, refer to Ulrych and Bishop (1975) and Strand (1977). The method had been adopted previously in our works of ionospheric disturbances analysis (Wan et al., 1998; Ding et al., 2007). A running time
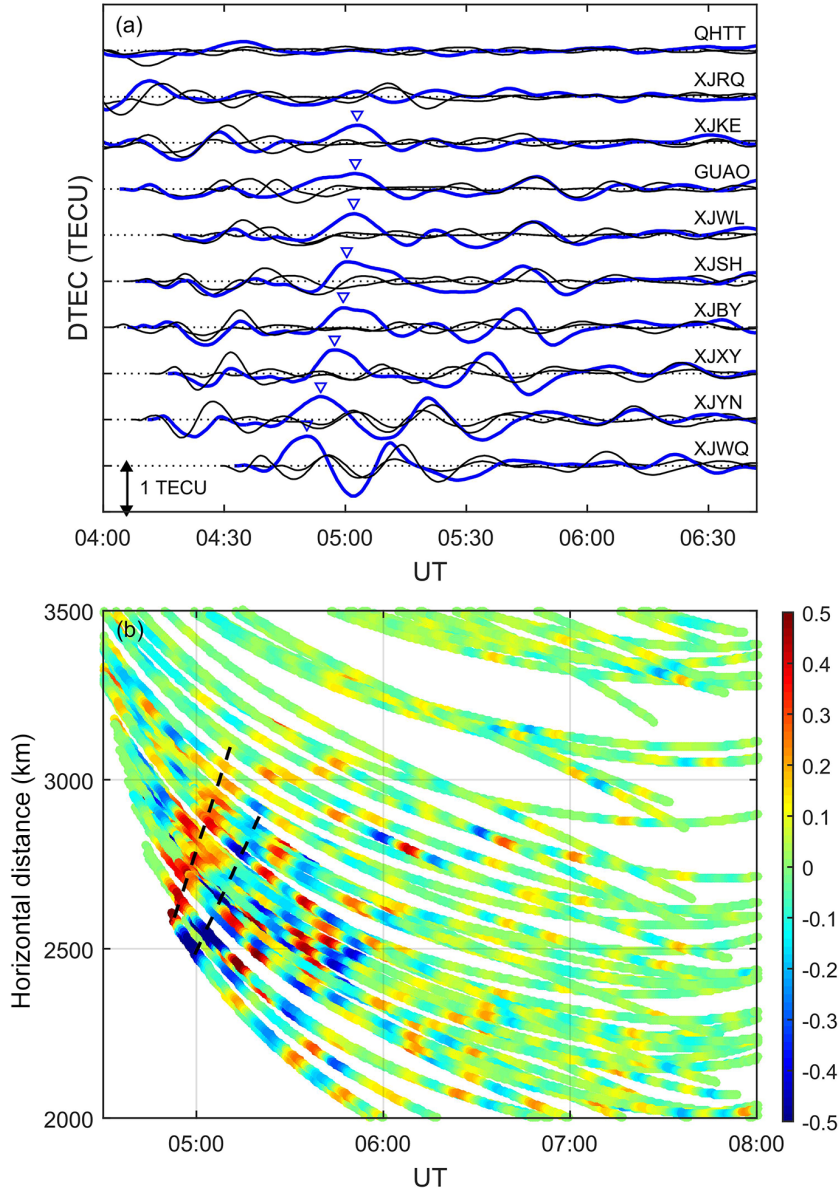

Figure 3. (a) Temporal and (b) spatial variations of the TEC perturbations observed by the GPS stations in northwest China on 15 February 2013. Panel (a) shows the series from 10 selected stations whose locations are marked by red and blue dots in Fig. 1. The codes of the stations are marked near each series. Blue lines in panel (a) represent the TEC perturbation series on 15 February 2013 and black lines the time series prior to and after that date. Triangles in panel (b) indicate the maximum of the TID's amplitude. Contours in panel (b) represent the amplitudes of the TEC perturbation in TECU units. The horizontal distance ( $y$ axis) in panel (b) is the spherical distance between the blast site and the ionospheric TEC pierce points at an altitude of $350 \mathrm{~km}$. Dotted lines in panels (ab) denote the phase fronts of the TID. All TEC perturbation series are from GPS satellite PRN 24.

window of $30 \mathrm{~min}$ was used during the calculation. A detailed introduction of this method can be found in Ding et al. $(2004,2011)$. It is shown in Fig. 4 that the propagation parameters of the second TID remains largely unchanged during 04:57-05:30 UT. The small variation of the wave parameters implies that there was a steady wave field pass by during this period. The average values of the period, velocity, and azimuth were generally consistent with those obtained from Fig. 3. 

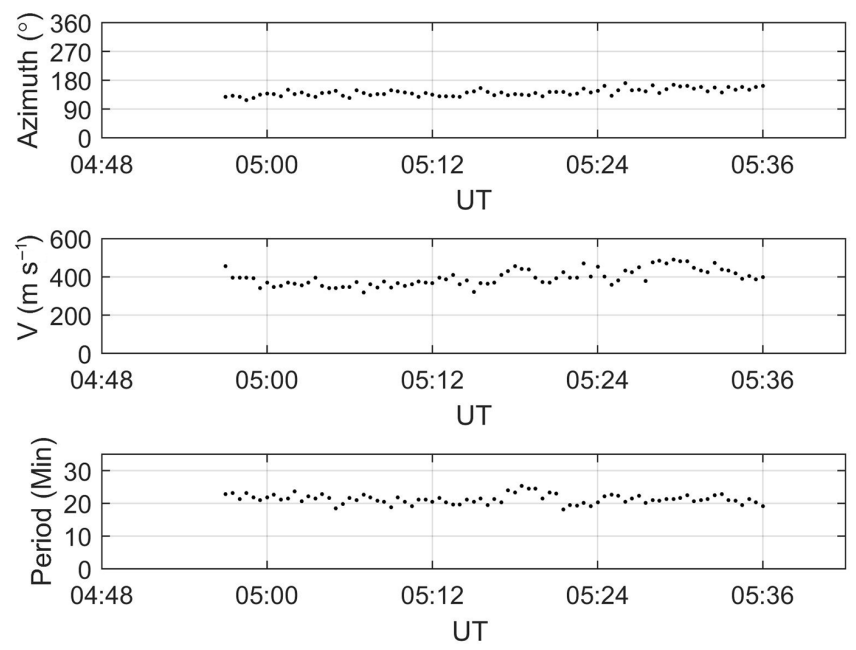

Figure 4. Temporal variations of propagation azimuth (clockwise from north, panel a), horizontal phase velocity (panel b), and period (panel c) for the traveling ionospheric disturbance observed on 15 February 2013 over northwest China. Temporal variations of the parameters were obtained by applying multi-channel maximum entropy method to the TEC time series observed by three Chinese GPS stations whose locations are marked as blue dots in Fig. 1.

\section{Discussion}

\subsection{Discussion on the properties of two TIDs following the meteorite blast}

Two TIDs were observed following the meteorite blast. The first TID was observed to propagate radially from the source with the range of at least $270-600 \mathrm{~km}$, and it was not noticed by the Chinese stations; the second one was observed at $\sim 2500-3100 \mathrm{~km}$ from the source, in northwest China. Although there is a dense GPS network in the east of China, no TIDs were observed there (not shown in figures).

The first TID we observed, which propagated with a period of $12 \mathrm{~min}$ and at a velocity of $369 \mathrm{~m} \mathrm{~s}^{-1}$, was first reported by Gokhberg et al. (2013), although they did not estimate its propagation parameters. Yang et al. (2014) also analyzed the GPS data from the ARTU station and addressed the same TID as we did. Generally, when a large explosion occurs, a shock wave expanding in all the directions can be expected. Such a radially propagating TID was observed in the present study (see Fig. 2c). The propagation range of the first TID seems to be limited to several hundred kilometers from the blast site. This may be because the dissipation of the TID's amplitude limits its propagation range. Although the dissipation of amplitude of the first TID cannot be directly observed because of the limited number of stations in the near-field of the blast site, strong dissipation of shock waves excited by other sources, such as rocket launches, has previously been addressed (Ding et al., 2014).
Note that the second TID was not observed by the ARTU station, the nearest GPS station to the blast site. The second TID discussed in the present study was previously observed elsewhere in other directions with respect to the blast site. Using the backscatter radar in Chelyabinsk, Berngardt et al. (2013) observed a TID that propagated at $1500-4000 \mathrm{~km}$ north of the blast site. The TID they observed was characterized by a period and a propagation velocity of $20 \mathrm{~min}$ and $350 \mathrm{~m} \mathrm{~s}^{-1}$, respectively. Based on observations by GPS receivers in central Asia, Yang et al. (2014) observed a southwards-propagating TID at $1500 \mathrm{~km}$ south of the blast site; they estimated its period and velocity at 24-60 min and $363 \mathrm{~m} \mathrm{~s}^{-1}$, respectively. The periods and velocities obtained by Berngardt et al. (2013) and Yang et al. (2014) are close to those we derived from our observations. This indicates that the second TID in the present study might have been propagated radially away from the blast site in different directions, across a much wider area than was observed. However, this does not allow us to interpret the second TID as having been excited directly by the blast in the near-field area of the blast site. As shown in Fig. 3b, the second TID was observed at $\sim 2500-3100 \mathrm{~km}$ from the meteorite blast site. If the TID had been propagated directly from the blast site, it would have caused TEC perturbations with large amplitude in the area within $2500 \mathrm{~km}$. However, although there were some observations of slant TEC whose ionospheric pierce points went across the area within $2500 \mathrm{~km}$, such TIDs were not observed within that range. Hence, our observation did not show any evidence that the second TID and the first one were the same event.

A possible interpretation of the second TID is that it may be a secondary wave excited by the first TID, which was observed by ARTU. As shown in the last section, the first and second TIDs had the maximum absolute amplitudes of 0.35 and 0.6 TECU, respectively. While the second TID had an absolute amplitude larger than that of the first TID, its relative amplitude was smaller than that of the first one. According to GIM data, during the time of the TID propagation, the background TEC is averaged at $\sim 13$ TECU at ARTU and $\sim 27$ TECU in northwest China. As the first and the second TIDs had absolute amplitudes of 0.35 and $0.6 \mathrm{TECU}$, their relative amplitudes were 2.7 and $2.2 \%$, respectively. Excitation of a secondary wave had been reported by Breitling and Kupferman (1967), who used globally distributed ionosondes to observe TIDs following nuclear detonations and found that the shock waves excited by the explosions might eventually degenerate into several waves that could be observed at large distances as TIDs. Using the GPS network, Calais and Minster (1996) analyzed the TIDs associated with a space shuttle ascent and concluded that the launch excited a shock wave, followed by secondary wave excited by the shock. Recently, Vadas and Liu (2009) used modeling to conclude that the dissipation of medium-scale gravity waves could generate secondary waves in the thermosphere, which is consistent 
with our interpretation. However, observational evidence for such an interpretation remains to be obtained.

\subsection{Comparison of TIDs excited by the meteorite blast with TIDs excited by nuclear explosions}

As regards the energy release, the Chelyabinsk meteorite's blast ( $\sim 500 \mathrm{kt}$ of TNT; Borovička et al., 2013) did not exceed the equivalent releases of most man-made nuclear explosions in the troposphere. Nuclear explosions that were detonated as weapons tests took place mainly in the 1950s and 1960s before the Partial Nuclear Test Ban Treaty was signed. During those years, atmospheric and ionospheric disturbances that were possibly excited by these explosions were studied extensively through observations of globally distributed microbarographs and ionosondes, respectively (Yamamoto, 1954; Donn and Ewing, 1962; Wickersham, 1966). As meteorite blasts happen only rarely, it is most convenient to compare our results with those early observations in the context of possible propagation mechanisms of the ionospheric disturbances.

According to the early observations, following nuclear explosions the microbarographs recorded acoustic waves in the air pressure with periods ranging from 0.5 to $9 \mathrm{~min}$ and velocities near the sound velocity. These waves propagated globally in the troposphere (Carpenter et al., 1961; Donn and Ewing, 1962). A thermal waveguide in the troposphere was invoked to account for this global propagation. In the ionosphere, TIDs with estimated velocities ranging widely from 50 to $900 \mathrm{~m} \mathrm{~s}^{-1}$ were observed as perturbations in the critical frequency of the F2 layer ( $f \circ \mathrm{F} 2$; Breitling and Kupferman, 1967). Note that the observations might be biased by the sparse geographical distribution of the ionosondes and the long sampling time intervals. The periods of the TIDs could not be identified because the ionosondes at that time routinely provided ionograms at time intervals of $1 \mathrm{~h}$. Alternatively, some authors compared their data with theoretical dispersion-curve results to estimate the ducted wave modes that could possibly support global TID propagation. These ducted modes were generally sustained by the large vertical temperature gradient at the bottom of the thermosphere (Hunsucker, 1982). A typical study was published by Wickersham (1966), who used the ionosonde network in Europe to monitor the ionospheric disturbances after a nuclear explosion equivalent to $50 \mathrm{Mt}$ of TNT at Novaya Zemlya on $30 \mathrm{Oc}-$ tober 1961. Wickersham (1966) observed TIDs at $\sim 1300$ $4400 \mathrm{~km}$ from the explosion site and proposed several acoustic ducted modes (with periods of less than $10 \mathrm{~min}$ ) as well as gravity-wave ducted modes (with longer periods) to account for the propagation mechanisms. However, Hines (1967) analyzed the same database and concluded that, concerning the oblique propagation of wave energy, the identification of acoustic modes was not validated due to contrast between observations and the theory, while the interpretation of the TIDs as gravity-wave modes was consistent with the observations.
These observations indicate that nuclear explosions could excite TIDs, which then probably propagated globally according to gravity-wave ducted modes. However, such global TID propagation was not observed following the Chelyabinsk meteorite blast in the present study. Two TIDs associated with gravity waves were observed after the blast. The first TID was observed to propagate at least $270-600 \mathrm{~km}$ from the source, and it was not noticed by the Chinese stations which are more than $1500 \mathrm{~km}$ away; the second one was observed at $\sim 2500-3100 \mathrm{~km}$ from the source, in northwest China. Neither of them have been observed by the dense GPS network or the ionosonde chain in the east of China (not shown in figures).

A reasonable explanation is that the energy release of the Chelyabinsk meteorite blast is not large enough to excite such global-scale ionospheric disturbances. The energy release of the nuclear explosions mentioned in the above reference ranges from 1.4 to $50 \mathrm{Mt}$ of TNT (see Table 4 of Breitling and Kupferman, 1967). This is much larger than the $500 \mathrm{kt}$ of energy release during the Chelyabinsk meteorite blast (Borovička et al., 2013).

Theoretical analysis also does not support the longdistance propagation of TIDs excited during meteorite blast. Given the observed periods and velocities, the TIDs we obtained were of medium scale. According to the simulations of Francis (1975) and Ding et al. (2003), while large-scale TIDs can propagate long distances in ducted modes, medium-scale TIDs always propagate as freely propagating internal waves and suffer more dissipation. Such severe dissipation of amplitudes can be seen in our results (Fig. 3a). An idealized ducted mode in the thermosphere requires that the background conditions remain unchanged during TID propagation. Considering the large temporal and spatial variations in a realistic thermosphere, medium-scale TIDs face more difficulty in propagating in a ducted mode than their largescale counterparts because of the low velocities and their long propagation durations. The dissipation of medium-scale TIDs that we measured has been predicted by the transferfunction modeling work of Mayr et al. (1990), who found that low-speed gravity waves with short periods are affected by increased energy attenuation. It is also implied from the above analysis that it may be the large-scale TIDs that were excited by the energetic nuclear explosions in 1950-1960s and caused the global perturbations in $f o \mathrm{~F} 2$.

Nevertheless, Yang et al. (2014) reported global TID propagation during the same meteorite blast event. Based on data from a dense GPS network in North America, Yang et al. (2014) observed a TID over North America $\sim 3 \mathrm{~h}$ after the blast. The TID propagated westwards with a period and velocity of 2-6 min and $733 \mathrm{~m} \mathrm{~s}^{-1}$, respectively. Such a TID was not observed after the blast elsewhere in the world, particularly not in those regions covered by dense GPS networks, such as China (the present work) and Japan (Yang et al., 2013). Although the global propagation of acoustic waves in the troposphere was observed by ground-based in- 
frasound sensors (Pichon et al., 2013), global-scale propagation of TIDs associated with acoustic waves in the ionosphere has not been reported to date. According to theoretical calculation, TIDs characterized by such scales would suffer from considerable dissipation and propagate in a limited range (Hines, 1967; Mayr et al., 1990). In fact, dissipation of acoustic waves in the ionosphere has been observed by Ding et al. (2014), who found that TIDs associated with acoustic waves excited by rocket launch have a maximum propagation range of no more than $1500 \mathrm{~km}$. Based on above analysis, the relation between the TID observed over North America (Yang et al., 2014) and the meteorite blast in Chelyabinsk remains to be identified.

\section{Summary}

This paper reports GPS-network observations of TIDs that were possibly excited by the large meteorite blast over Chelyabinsk, Russia, on 15 February 2013. The observations were conducted through 75 GPS stations in northwest China and central Asia. We observed two TIDs, which occurred $13 \mathrm{~min}$ and $1.5 \mathrm{~h}$ after the meteorite blast. The first TID was observed $270-600 \mathrm{~km}$ from the blast site, propagating radially from the site with a mean velocity and period of $369 \mathrm{~m} \mathrm{~s}^{-1}$ and $12 \mathrm{~min}$, respectively. The second TID was observed in northwest China $1.5 \mathrm{~h}$ after the meteorite blast, at $\sim 2500-3100 \mathrm{~km}$ from the blast site. This latter TID propagated southeastwards with a velocity and period of $410 \mathrm{~m} \mathrm{~s}^{-1}$ and $23 \mathrm{~min}$, respectively. During the passage of the TID, severe dissipation of the TEC perturbation amplitudes was observed. The second TID was previously observed elsewhere in other directions. This indicates that the TID might propagate radially away from the blast site in different directions, across a much wider area than was observed.

We did not find any TIDs propagating in a global range after the meteorite blast. This is different from early results that TIDs propagated globally following nuclear explosions. Because the energy release of the Chelyabinsk meteorite blast $(\sim 500 \mathrm{kt}$ of TNT) was considerably smaller than those of the nuclear explosions (1.4 to $50 \mathrm{Mt}$ of TNT), we estimated that the energy release of the Chelyabinsk meteorite blast might not be large enough to excite such ionospheric disturbances in a global range as some nuclear explosions have done.

\section{Data availability}

GPS data used in this article consist of data from the IGS and data from the GPS network of the China Meteorological Administration (CMA). One can acquire the former from http://cddis.gsfc.nasa.gov (CDDIS, 2016). To access the latter, please contact the second author of this article (Tian Mao, email address: maotian@cma.gov.cn).
Acknowledgements. We acknowledge the use of data from the the GPS network of the China Meteorological Administration (CMA), the International GNSS Service (IGS), and the Chinese Meridian Project. The GIM data used in this study were acquired as part of NASA's Earth Science Data Systems and archived and distributed by the Crustal Dynamics Data Information System (CDDIS). This work was supported by the National Natural Science Foundation of China (grants 41574151, 41274162, 41204113 and 41321003), and the National High Technology Research and Development Program of China (863 Program) (no. 2014AA123503).

The topical editor, H. Kil, thanks I. Kutiev and one anonymous referee for help in evaluating this paper.

\section{References}

Berngardt, O. I., Kurkin, V. I., Zherebtsov, G. A., Kusonski, O. A., and Grigorieva, S. A.: Ionospheric effects during first 2 hours after the Chelyabinsk meteorite impact, arXiv1:1308.3918 [physics.geo-ph], available at: https://arxiv. org/pdf/1308.3918v1.pdf, 2013.

Borovička, J., Spurný, P., Brown, P., Wiegert, P., Kalenda, P., Clark, D., and Shrbený, L.: The trajectory, structure and origin of the Chelyabinsk asteroidal impactor, Nature, 503, 7475, 235-237, doi:10.1038/nature12671, 2013.

Breitling, W. J. and Kupferman, R. A.: Traveling ionospheric disturbances associated with Nuclear Detonations, J. Geophys. Res., 72, 307-315, 1967.

Brown, P.: A preliminary report on the Chelyabinsk fireball/airburst, WGN, the Journal of the IMO, 41, available at: http://meteor. uwo.ca/publications/wgn-chel.pdf.pdf, 2013.

Calais, E. and Minster, J. B.: GPS detection of ionospheric perturbations following a Space Shuttle ascent, Geophys. Res. Lett., 23, 1897-1900, 1996.

Carpenter, E. W., Harwood, G., and Whiteside, T.: Microbarograph records from the Russian large nuclear explosions, Nature, 4805, p. $857,1961$.

CDDIS: Crustal Dynamics Data Information System (CDDIS) of the National Aeronautics and Space Administration (NASA), Receiver Independent Exchange Format (RINEX) files, available at: http://cddis.gsfc.nasa.gov, 2016.

Ding, F., Wan, W., and Yuan, H.: The influence of background winds and attenuation on the propagation of atmospheric gravity waves, J. Atmos. Sol.-Terr. Phy., 65, 857-869, doi:10.1016/S1364-6826(03)00090-7, 2003.

Ding, F., Yuan, H., Wan, W., Reid, I. M., and Woithe, J. M.: Occurrence characteristics of medium-scale gravity waves observed in $\mathrm{OH}$ and $\mathrm{OI}$ nightglow over Adelaide $\left(34.5^{\circ} \mathrm{S}, 138.5^{\circ} \mathrm{E}\right)$, J. Geophys. Res., 109, D14104, doi:10.1029/2003JD004096, 2004.

Ding, F., Wan, W., Ning, B., and Wang, M.: Large-scale traveling ionospheric disturbances observed by GPS total electron content during the magnetic storm of 29-30 October 2003, J. Geophys. Res., 112, 309, doi:10.1029/2006JA012013, 2007.

Ding, F., Wan, W., Xu, G., Yu, T., Yang, G., and Wang, J.: Climatology of medium-scale traveling ionospheric disturbances observed by a GPS network in central China, J. Geophys. Res., 116, A09327, doi:10.1029/2011JA016545, 2011.

Ding, F., Wan, W., Mao, T., Wang, M., Ning, B., Zhao, B., and Xiong, B.: Ionospheric response to the shock and acoustic waves 
excited by the launch of the Shenzhou 10 spacecraft, Geophys. Res. Lett., 41, 3351-3358, doi:10.1002/2014GL060107, 2014.

Donn, W. L. and Ewing, M.: Atmospheric waves from nuclear explosions, J. Geophys. Res., 67, 1855-1866, 1962.

Francis, S. H.: Global propagation of atmospheric gravity waves: A review, J. Atmos. Terr. Phy., 37, 1011-1030, 1975.

Gokhberg, M. B., Ol'shanskaya, E. V., Steblov, G. M., and Shalimov, S. L.: The Chelyabinsk Meteorite: Ionospheric Response Based on GPS Measurements, Dokl. Earth Sci., 452, 948-952, 2013.

Hines, C. O.: On the Nature of traveling ionospheric disturbances launched by low-altitude nuclear explosions, J. Geophys. Res., 72, 1877-8882, 1967.

Hunsucker, R. D.: Atmospheric gravity waves generated in the high-latitude ionosphere: A review, Rev. Geophys., 20, 293-315, doi:10.1029/RG020i002p00293, 1982.

Mayr, H. G., Harris, I., Herrero, F. A., Spencer, N. W., Varosi, F., and Pesnell, W. D.: Thermospheric gravity waves: observations and interpretation using the transfer function model (TFM), Space Sci. Rev., 54, 297-375, 1990.

Noll, C.: The Crustal Dynamics Data Information System: A resource to support scientific analysis using space geodesy, Adv. Space Res., 46, 1421-1440, doi:10.1016/j.asr.2010.01.018, 2012.

Pichon, A. L., Ceranna, L., Pilger, C., Mialle, P., Brown, D., Herry, P., and Brachet, N.: The 2013 Russian fireball largest ever detected by CTBTO infrasound sensors, Geophys. Res. Lett., 40, 3732-3737, doi:10.1002/grl.50619, 2013.
Popova, O. P., Jenniskens, P., Emelýanenko, V., Kartashova, A., Biryukov, E. et al.: Chelyabinsk Airburst, Damage Assessment, Meteorite Recovery, and Characterization, Science, 342, 1069, doi:10.1126/science.1242642, 2013.

Strand, O.: Multichannel complex maximum entropy (autoregressive) spectral analysis, IEEE Trans. Automat. Contr., 22, 634640, doi:10.1109/TAC.1977.1101545, 1977.

Ulrych, T. J. and Bishop, T. N.: Maximum entropy spectral analysis and autoregressive decomposition, Rev. Geophys., 13, 183, doi:10.1029/RG013i001p00183, 1975.

Vadas, S. L. and Liu, H.: Generation of large-scale gravity waves and neutral winds in the thermosphere from the dissipation of convectively generated gravity waves, J. Geophys. Res., 114, A10310, doi:10.1029/2009JA014108, 2009.

Wan, W. X., Yuan, H., Ning, B., and Liang, J.: Travelling ionosphere disturbances associated with tropospheric vortexes around Qinghai-Tibet Plateau, Geophys. Res. Lett., 25, 3775-3778, doi:10.1029/1998GL900030, 1998.

Wickersham Jr., A. F.: Identification of acoustic gravity wave modes from ionospheric range time observations, J. Geophys. Res., 71, 4551-4555, 1966.

Yamamoto, R.: The microbarographic oscillations produced by the explosions of hydrogen-bombs, Bulletin of the Institute for Chemical Research, Kyoto University, 32, 120-133, 1954.

Yang, Y.-M., Komjathy, A., Langley, R. B., Vergados, P., Butala, M. D., and Mannucci, A. J.: The 2013 Chelyabinsk meteor ionospheric impact studied using GPS measurements, Radio Sci., 49, 341-350, doi:10.1002/2013RS005344, 2014. 